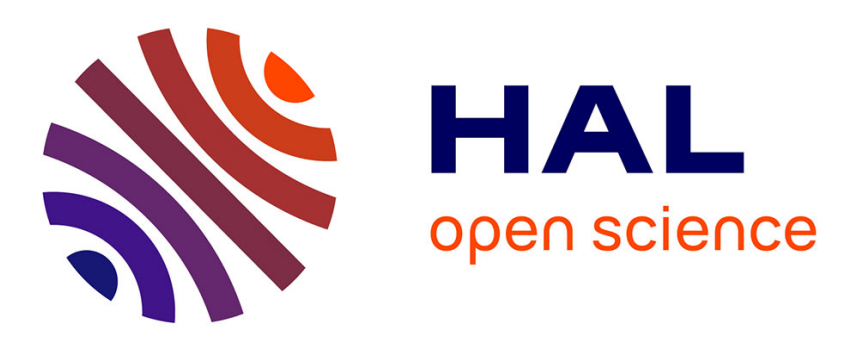

\title{
Application of a combined finite element -finite volume method to a 2D non-hydrostatic shallow water problem
}

Nora Aissiouene, Marie-Odile Bristeau, Edwige Godlewski, Anne Mangeney, Carlos Parés, Jacques Sainte-Marie

\section{- To cite this version:}

Nora Aissiouene, Marie-Odile Bristeau, Edwige Godlewski, Anne Mangeney, Carlos Parés, et al.. Application of a combined finite element -finite volume method to a 2D non-hydrostatic shallow water problem. FVCA 8, Jun 2017, Lille, France. pp.219-226. hal-01664481

\section{HAL Id: hal-01664481 https://hal.science/hal-01664481}

Submitted on 14 Dec 2017

HAL is a multi-disciplinary open access archive for the deposit and dissemination of scientific research documents, whether they are published or not. The documents may come from teaching and research institutions in France or abroad, or from public or private research centers.
L'archive ouverte pluridisciplinaire HAL, est destinée au dépôt et à la diffusion de documents scientifiques de niveau recherche, publiés ou non, émanant des établissements d'enseignement et de recherche français ou étrangers, des laboratoires publics ou privés. 


\title{
Application of a combined finite element - finite volume method to a $2 \mathrm{D}$ non-hydrostatic shallow water problem
}

\author{
N. Aïssiouene, M-O. Bristeau, E. Godlewski, \\ A. Mangeney, C. Parés and J. Sainte-Marie
}

June 2017

\begin{abstract}
We propose a numerical method for a two-dimensional non-hydrostatic shallow water system with topography [6]. We use a prediction-correction scheme initially introduced by Chorin-Temam [13], and which has been applied previously to the one dimensional problem in [1]. The prediction part leads to solving a shallow water system for which we use finite volume methods [3], while the correction part leads to solving a mixed problem in velocity/pressure using a finite element method. We present an application of the method with a comparison between a hydrostatic and a non-hydrostatic model.
\end{abstract}

\section{Introduction}

Mathematical models for free surface flows are widely studied, however one still needs to improve the existing models as well as develop robust numerical methods. The most common way to represent the physical behavior of the free surface is to compute the solutions of the Shallow Water equations. These equations are based on a shallowness assumption and lead to assuming the pressure is hydrostatic. Therefore, they are used for many geophysical flows on rivers, lakes, oceans where the characteristic horizontal length is much greater than the depth.

However, when the hydrostatic assumption is no longer valid, what we call dispersive effects appear, then more complex models have to be used to represent these effects. Many free surface models are available to take into consideration this dispersive effect, see [11] for the classical Green-Naghdi (GN) model and [5, 7, 9, 6] for other kinds of non hydrostatic models with bathymetry.

In this approach, we propose a new method dealing with a formulation without high order terms, we treat the depth-averaged Euler system developed in [6] where the non-hydrostatic pressure is an unknown of the system. The aim is to provide a robust numerical method for the two-dimensional model on an unstructured grid. The objective is to have a stable method to simulate real cases where the topography can be complex and needs an irregular mesh. Moreover, it gives the possibility to perform adaptive meshes if one wants to refine the mesh in the areas where the dispersive effects are expected. For instance, the dispersive contribution can have 


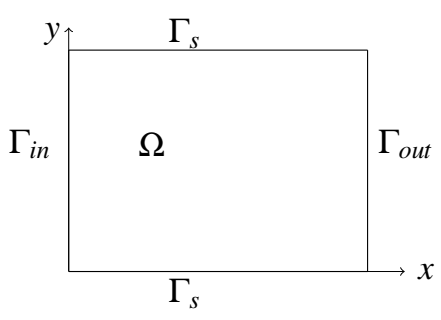

(a) View from above.

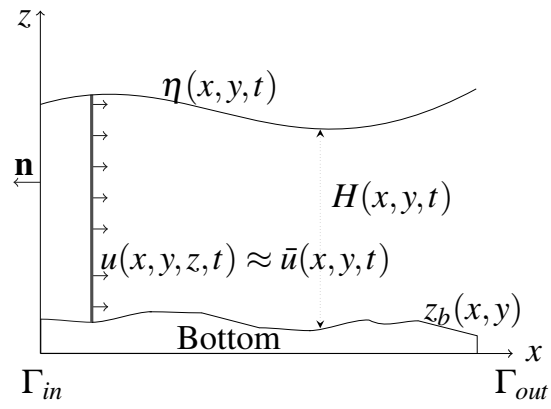

(b) Vertical cross section.

Figure 1: Model domain and notations.

a significant impact in the water depth for the propagation of tsunamis [10],[4].

The paper is organized as follows. In the next section, we recall the depth-averaged Euler system. The Section 2 is devoted to the Chorin-Temam approach (predictioncorrection scheme) applied for the model problem, while in Section 4, we give a geophysical application where we compare the results using a hydrostatic model vs a non-hydrostatic model.

\section{The averaged Euler system}

We consider a two-dimensional domain $\Omega \subset \mathbb{R}^{2}$ delimited by the boundary $\Gamma=$ $\Gamma_{\text {in }} \cup \Gamma_{\text {out }} \cup \Gamma_{s}$ as described in Figure 1a. We denote by $H(x, y, t)$ the water depth, $z_{b}(x, y)$ the topography, $\mathbf{u}(x, y, t)$ the averaged velocity of the fluid $\mathbf{u}=(u, v, w)^{t}$ and $p$ the non hydrostatic pressure (see Figure $1 \mathrm{~b}$ ).

The two-dimensional depth-averaged Euler system described in [6] reads:

$$
\begin{aligned}
\frac{\partial H}{\partial t}+\nabla_{0} \cdot(H \mathbf{u}) & =0 \\
\frac{\partial H \mathbf{u}}{\partial t}+\nabla_{0} \cdot(H \mathbf{u} \otimes \mathbf{u})+\nabla_{0}\left(\frac{g}{2} H^{2}\right)+\nabla_{s w}(p) & =-g H \nabla_{0}\left(z_{b}\right), \\
\operatorname{div}_{s w}(\mathbf{u}) & =0
\end{aligned}
$$

where we define the operators $\nabla_{0}$ and $\operatorname{div}_{0}$ by

$$
\nabla_{0} f=\left(\begin{array}{c}
\frac{\partial f}{\partial x} \\
\frac{\partial f}{\partial y} \\
0
\end{array}\right), \quad \operatorname{div}_{0} \mathbf{v}=\frac{\partial v_{1}}{\partial x}+\frac{\partial v_{2}}{\partial y}
$$

Also, we give an interpretation of the non-hydrostatic contribution by defining a shallow water version of the pressure gradient $\nabla_{s w}$ and the divergence operator 
$\operatorname{div}_{s w}$. Assuming that $f$ and $\mathbf{v}=\left(v_{1}, v_{2}, v_{3}\right)^{T}$ are smooth enough:

$$
\begin{aligned}
\nabla_{s w} f & =\left(\begin{array}{c}
H \frac{\partial f}{\partial x}+f \frac{\partial\left(H+2 z_{b}\right)}{\partial x} \\
H \frac{\partial f}{\partial y}+f \frac{\partial\left(H+2 z_{b}\right)}{\partial y} \\
-2 f
\end{array}\right), \\
\operatorname{div}_{s w}(\mathbf{v}) & =\frac{\partial H v_{1}}{\partial x}+\frac{\partial H v_{2}}{\partial y}-v_{1} \frac{\partial\left(H+2 z_{b}\right)}{\partial x}-v_{2} \frac{\partial\left(H+2 z_{b}\right)}{\partial y}+2 v_{3} .
\end{aligned}
$$

Under the assumptions done for the derivation of the non-hydrostatic model, the operator $\nabla_{s w}$ (resp. $\operatorname{div}_{s w}$ ) is the average of the classical operator $\nabla$ (resp. div) in the sens that it corresponds to the gradient averaged in the vertical direction between $z_{b}$ and $\eta$. An important property is that the operators $\operatorname{div}_{s w}$ and $\nabla_{s w}$ satisfy the duality relation

$$
\int_{\Omega} \nabla_{s w}(f) \cdot \mathbf{v}=-\int_{\Omega} \operatorname{div}_{s w}(\mathbf{v}) f+\int_{\Gamma} H f \mathbf{v} \cdot \mathbf{n},
$$

where $\mathbf{n}$ is the outward unit normal vector to the boundary $\Gamma$. This property is crucial for the algorithm presented in the following since we will consider a mixed problem in velocity/pressure, which will lead, at the numerical level, to having an operator for the pressure and its transpose for the velocity.

The depth-averaged model (1)-(3) is derived in [6] and is based on the minimization of the energy (see [12], this property provides a consistency with the Euler system [6] in terms of energy.

Notice that we consider the non-hydrostatic pressure $p$ as an unknown of the model, but we can write the total pressure $p_{\text {tot }}$ as:

$$
p_{\text {tot }}=g \frac{H}{2}+p,
$$

where we take into account the hydrostatic pressure $g \frac{H}{2}$.

\section{Prediction - Correction scheme}

The problem (1)-(3) is solved using a Chorin-Temam splitting scheme (see [13]). The prediction-correction method is widely used to approximate the Navier-Stokes equations and is based on a time-splitting scheme. For each time step, the problem is solved in two steps, in the first one, we use a finite-volume method to solve the hyperbolic part which is a Shallow Water system with topography (where the non hydrostatic pressure $p$ is not evaluated). This allows us to get a first predicted state which is not divergence free. In the second step, we update the predicted state with the shallow water version of the gradient pressure evaluated in such a way that the velocity satisfies the divergence free condition (3).

Let us denote by $X$ the vectors of unknowns and $F(X)$ the matrix:

$$
X=\left(\begin{array}{c}
H \\
H u \\
H v \\
H w
\end{array}\right), \quad F(X)=\left(\begin{array}{cc}
H u & H v \\
H u^{2}+\frac{g}{2} H^{2} & H u v \\
H u v & H v^{2}+\frac{g}{2} H^{2} \\
H u w & H v w
\end{array}\right) \text {, }
$$


and set

$$
S(X)=\left(\begin{array}{c}
0 \\
-g H \frac{\partial z_{b}}{\partial x} \\
-g H \frac{\partial z_{b}}{\partial y} \\
0
\end{array}\right) \text { and } R_{n h}=\left(\begin{array}{c}
0 \\
\nabla_{s w}(p)
\end{array}\right)
$$

Then, the system (1)-(3) can be written

$$
\begin{gathered}
\frac{\partial X}{\partial t}+\operatorname{div}_{0} F(X)+R_{n h}=S(X), \\
\operatorname{div}_{s w}(\mathbf{u})=0 .
\end{gathered}
$$

We set $t^{0}$ the initial time and $t^{n+1}=t^{n}+\Delta t^{n}$ where $\Delta t^{n}$ satisfies a stability condition (CFL) and the state $X^{n}$ will denote an approximation of $X\left(t^{n}\right)$. For each time step, we consider an intermediate state which will be denoted with the superscript $n+1 / 2$. The semi discretization in time can be summarized in the following steps:

$$
\begin{aligned}
X^{n+1 / 2} & =X^{n}-\Delta t^{n} \operatorname{div}_{0} F\left(X^{n}\right)+\Delta t S\left(X^{n}\right), \\
X^{n+1}+\Delta t^{n} R_{n h}^{n+1} & =X^{n+1 / 2}, \\
\operatorname{div}_{s w} \mathbf{u}^{n+1} & =0 .
\end{aligned}
$$

So the first step (13) leads to solving the hyperbolic system with source terms in order to get the state $X^{n+1 / 2}=\left(H^{n+1 / 2},(H u)^{n+1 / 2},(H v)^{n+1 / 2},(H w)^{n+1 / 2}\right)^{T}$. Equation (14) allows us to correct the predicted value $X^{n+1 / 2}$ in order to obtain a state which satisfies the divergence free condition (15).

The prediction part (13) is solved using a cell centered finite-volume method [3]. For this system, our scheme is second order accurate in time and, if we use a reconstruction algorithm [3] in the hyperbolic step, it is formally second order accurate in space $[3,2]$. In the application, we use a kinetic solver for its good mathematical properties. The correction part (14) is solved using a finite element method. To do so, we consider the equations (14)-(15) as a mixed problem [2] and, starting with an appropriate variational formulation of the problem, we apply the finite element method to obtain the pressure $p^{n+1}$ which is solution of an elliptic equation and the velocity $\mathbf{u}^{n+1}$. The elliptic equation of the pressure can be written under the form:

$$
\operatorname{div}_{s w}\left(\frac{\nabla_{s w} p^{n+1}}{H^{n+1}}\right)=\frac{1}{\Delta t^{n}} \operatorname{div}_{s w}\left(\frac{(H \mathbf{u})^{n+1 / 2}}{H^{n+1 / 2}}\right)
$$

We consider a primal mesh which is the triangular mesh and a dual mesh corresponding to the centered finite volume cells. The approximation of the variables is based on the triangular mesh for the finite element scheme and the dual mesh for the finite volume scheme. The finite volume cells are centered on the vertices and built by joining the centers of mass of the triangles surrounding each vertex. The variables $H, H \mathbf{u}$ are estimated first as constant mean values on the cells by the finite volume scheme, which gives the intermediate state $X^{n+1 / 2}$. For the finite element scheme, the state $X^{n+1}$ is approximated at the vertices of the triangles. The algorithm uses an iterative method of Uzawa type to solve the elliptic equation in pressure involved in the problem. The details of the combined method and the treatment of the boundary conditions will be detailed in a forthcoming paper. 


\section{Numerical results}

In this section we test the depth-averaged model (1)-(3) on a numerical application. We generate small amplitude waves at the inlet of a domain of dimensions $[0,10] \times$ $[0,6]$ and we observe the propagation of the waves over an obstacle. The channel is also ended by a slope of $40 \%$. This simulation allows us to confront our method to a test case where we have a variable bottom with strong variations of the elevation and wet/dry interfaces. The dimensions of the case are described in Figure 2 and the obstacle is defined by the topography function:

$$
z_{b}(x, y)=\min \left(z_{m}, A e^{-\left(\left(a\left(x-x_{0}\right)^{2}\right)+b\left(y-y_{0}\right)^{2}\right)}\right),
$$

where we set $z_{m}=0.5 m, A=2 m, a=3.3 m, b=1.51 m$ and $x_{0}=3 m, y_{0}=3 m$. We set an initial free surface $\eta_{0}=0.6 \mathrm{~m}$ and a sinusoidal wave given at the inlet with an amplitude of $0.02 \mathrm{~m}$. The test is performed over an unstructured mesh of 45506 nodes for the fine mesh. The numerical solution is computed with a P1iso-P2/P1 approximation (see [1] for more details on the choices of approximation spaces). We compare the solutions obtained using the Shallow Water model and using the depth-averaged Euler model (1)-(3) in order to observe the effects of the dispersion on the propagation and the wave interactions. Figure 3 shows the simulations at instant $t_{1}=4.54531 \mathrm{~s}$ ( $3 \mathrm{a}$ and $3 \mathrm{~b}$ ) for the Shallow Water model (left) and the dispersive model (right). The figures represent the free surface $\eta$. We clearly observe the impact of the dispersive effects around the obstacle and on the forms of the waves. In Figure 4 we show the free surface over the time at different points around the obstacle and compare the solution obtained for the Shallow Water model and the depth averaged model. We can recover the same kind behavior in one dimensions for a very classical test case which is known as the Dingemans experiment [8], these effects occur when we have a strong variation of the topography with a a strong gradient of the elevation.

\section{Conclusion}

In this paper, we have presented an application a the combined finite-volume/ finite element method for a two dimensional dispersive shallow water model on an unstructured mesh. We solve a mixed problem using a finite element method to obtain the velocity and the non-hydrostatic pressure.

\section{Acknowledgments}

The authors acknowledge the Inria Project Lab Algae in Silicio for its financial support. The first author received a partial grant from the Fondation Ledoux. This research is also supported by the ANR MIMOSA project.

\section{References}

[1] Aïssiouene, N.: Numerical analysis and discrete approximation of a dispersive shallow water model. Theses, Pierre et Marie Curie, Paris VI (2016). URL https://hal.archives-ouvertes.fr/tel-01418676 


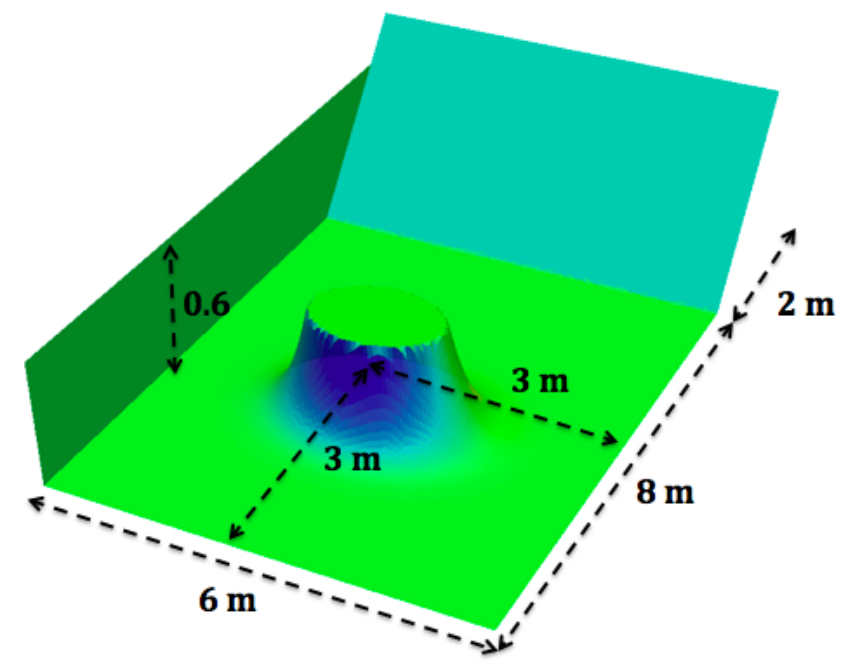

Figure 2: Dimension of the test case

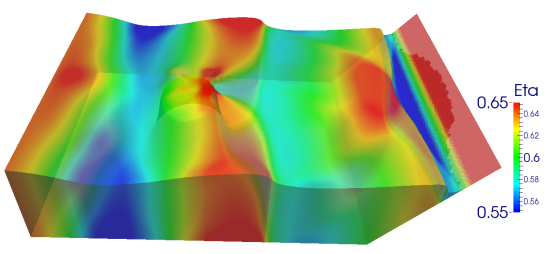

(a) Hydrostatic simulation at time $t_{1}=4.54531 \mathrm{~s}$

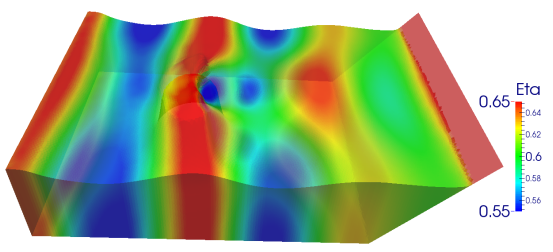

(b) Non-hydrostatic simulation at time $t_{1}$

Figure 3: Free surface obtained with a hydrostatic simulation and a non-hydrostatic simulation 


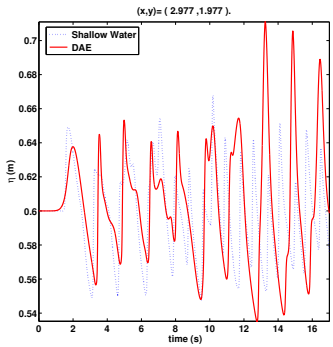

(a) Point 1

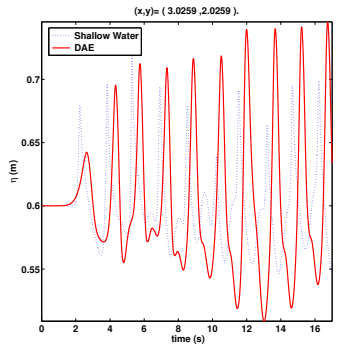

(b) Point 2

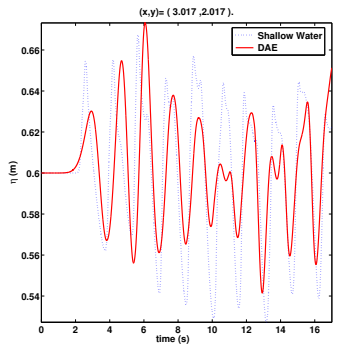

(c) Point 3

Figure 4: Comparison of the free surface over the time for the selected points between solutions computed with a hydrostatic model $(\cdots)$ and the depth-averaged model (-).

[2] Aïssiouene, N., Bristeau, M.O., Godlewski, E., Sainte-Marie, J.: A combined finite volume - finite element scheme for a dispersive shallow water system. Networks and Heterogeneous Media 11(1), 1-27 (2016). DOI 10.3934/nhm.2016.11.1. URL https://hal.inria.fr/hal-01160718

[3] Audusse, E., Bristeau, M.O.: A well-balanced positivity preserving secondorder scheme for Shallow Water flows on unstructured meshes. J. Comput. Phys. 206(1), 311-333 (2005)

[4] Behrens, J., Dias, F.: New computational methods in tsunami science. Philos Trans A Math Phys Eng Sci. Oct 28(373), (2053) (2015)

[5] Bonneton, P., Barthelemy, E., Chazel, F., Cienfuegos, R., Lannes, D., Marche, F., Tissier, M.: Recent advances in Serre-Green Naghdi modelling for wave transformation, breaking and runup processes. European Journal of Mechanics - B/Fluids 30(6), 589 - 597 (2011). DOI 10.1016/j.euromechflu.2011.02.005. URL http://www.sciencedirect.com/science/article/pii/S0997754611000185. Special Issue: Nearshore Hydrodynamics

[6] Bristeau, M.O., Mangeney, A., Sainte-Marie, J., Seguin, N.: An energy-consistent depth-averaged Euler system: Derivation and properties. Discrete and Continuous Dynamical Systems - Series B 20(4), 961-988 (2015). DOI 10.3934/dcdsb.2015.20.961. URL http://aimsciences.org/journals/displayArticlesnew.jsp?paperID=10801

[7] Chazel, F., Lannes, D., Marche, F.: Numerical simulation of strongly nonlinear and dispersive waves using a Green-Naghdi model. J. Sci. Comput. 48(1-3), 105-116 (2011). DOI 10.1007/s10915-010-9395-9. URL http://dx.doi.org/10.1007/s10915-010-9395-9

[8] Dingemans, M.W.: Wave propagation over uneven bottoms. Advanced Series on Ocean Engineering - World Scientific (1997)

[9] Duran, A., Marche, F.: Discontinuous-Galerkin discretization of a new class of Green-Naghdi equations. Communications in Computational Physics p. 130 (2014). URL https://hal.archives-ouvertes.fr/hal-00980826 
[10] Glimsdal, S., Pedersen, G.K., Harbitz, C.B., Løvholt, F.: Dispersion of tsunamis: does it really matter? Natural Hazards and Earth System Sciences 13(6), 1507-1526 (2013). DOI 10.5194/nhess-13-1507-2013. URL http://www.nat-hazards-earth-syst-sci.net/13/1507/2013/

[11] Green, A., Naghdi, P.: A derivation of equations for wave propagation in water of variable depth. J. Fluid Mech. 78, 237-246 (1976)

[12] Levermore, C., Sammartino, M.: A shallow water model with eddy viscosity for basins with varying bottom topography. Nonlinearity 14(6), 1493-1515 (2001)

[13] Rannacher, R.: On Chorin's projection method for the incompressible Navier-Stokes equations. In: G. Heywood John, K. Masuda, R. Rautmann, A. Solonnikov Vsevolod (eds.) The Navier-Stokes Equations II - Theory and Numerical Methods, Lecture Notes in Mathematics, vol. 1530, pp. 167183. Springer Berlin Heidelberg (1992). DOI 10.1007/BFb0090341. URL http://dx.doi.org/10.1007/BFb0090341 